\title{
Optimization of Gait Parameters for Energy Efficiency Improvement of Farmland Robot Based on Orthogonal Experiment Design
}

\author{
Yongnian ZHANG, Xiaofei LUO, Cong ZHU, Wei LU, Xiaochan WANG
}

\begin{abstract}
In order to improve the energy efficiency of legged robots while walking on farmland, a simulation system of legged robot on farmland soil was put forward in this paper. Firstly, the finite element model of the interaction between the robot leg and the farmland soil was established, and the multi-body dynamics and the foot-ground interaction simulation were carried out in one gait cycle. Secondly, the orthogonal experiment with four factors was established to analyze the external energy required for the robot leg to walk in the soil. Then the range analysis and variance analysis of the results were carried out to optimize the energy consumption ratio. The influence of the four factors on the experimental results and the optimal gait parameters and foot structure were obtained subsequently. Finally, the no-load walking experiment in the selfdeveloped soil-bin test-bed was carried out with the optimal gait and foot structure. The results demonstrate that the successive order of factors affecting the energy consumption ratio is foot structure, stride length, gait cycle time and stride height. The process of penetrating the soil consumes the most energy in a gait cycle. The optimization design of the foot structure and the penetration process is helpful to reduce the energy consumption of the farmland robot.
\end{abstract}

Keywords: legged robot; farmland soil; multi-body dynamics; orthogonal experiment; soil-bin test-bed

\section{INTRODUCTION}

Soil compaction will lower crop yields which is an increasingly challenging problem [1] in agricultural production and the effective way to mitigate the problem is adopting the legged robot [2-3]. The legged robot is becoming one of the hotspots and research directions in the field of robot with its incomparable advantages [4-6].

The legged robot is popularized [7] in agriculture especially in farmland information collection in America, Japan and other countries in the worldwide. A severe subsidence occurs [8] when the robot walks in the farmland where $80 \%$ of cultivated land is the paddy with deeper soft soil, lower bearing ability and shearing strength in southern region which is the major grain producing area. The solution to walking problem of legged robot in paddy field is of great significance to promote the development of farmland legged robot in China.

The robot has the ability to move in different environments depending on the interaction between the robot leg and the environment medium. At present there are few technical studies on the movement of legged robots in the farmland soils, and most of them focus on structure research, such as Zhang, Ren [9-10] studied the locomotion performance of the propulsion unit equipped with arched feet in the sandy and muddy terrain, the researchers in Jilin University have developed a series of unconventional walking mechanism. Zhang [11-12] analyzed the general terrain adaptability of the feet of ruminants using kinematics of the equivalent mechanism model based on screw theory. Ding [13] analyzed the foot-terrain interaction based on the knowledge of terramechanics and reveals the relationship between the parameters of the conventional models and the terramechanics models, but the subjects studied did not include farmland soil.

Just carrying on the bionic test cannot guarantee it is always applicable to all kinds of legged robot due to the differences in the structures, driving modes and degree of freedom between the animal and the legged robot. As CAD and CAE technologies have developed rapidly and the constitutive mode of soil gets better, the FEA method [1415] has become an effective way to analyze the soil mechanics problems. In this paper, a simulation system of legged robot on farmland soil is established to obtain the optimal gait parameters and foot structure for energy efficiency improvement.

\section{DESIGN OF THE ROBOT LEG}

To reduce the inertia of the legs and improve the dynamic characteristics of the robot, the lightweight materials should be used to reduce the weights and the drive motors should be arranged on the robot body in the optimal fashion [16]. This paper introduces a novel electric drive robot leg based on the double four-bar mechanism, as shown in Fig. 1. The positions fixing the hip and knee motors by bolting to the robot body are noted as $A$ and $D$, respectively. $D H, H I$, and $I K$ are the humerus, radius and foot, respectively, and a parallel four-bar linkage GHIJ is adopted to guarantee that the humerus and foot are kept parallel and increase the leg stiffness. $A B C D$ constitutes a crank rocker mechanism, and $D E F G$ constitutes a doublerocker mechanism. These two four-bar linkages drive the movement of the robot humerus and radius.

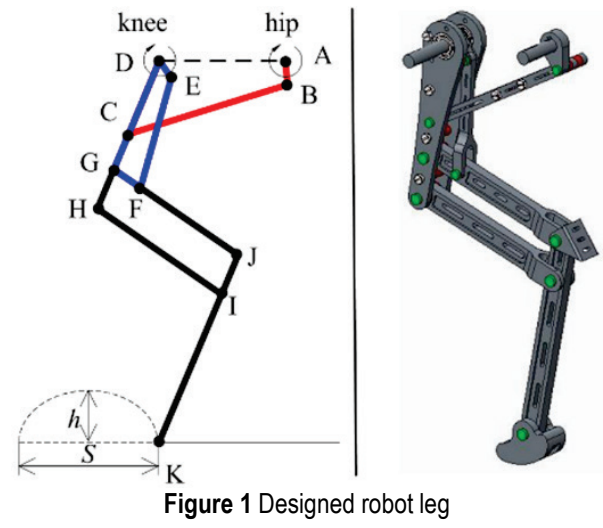

$A B C D$ : Compared with the knee motor, the hip motor needs to bear a greater load, and the frequent shifting between acceleration and deceleration will have a greater impact. Therefore, a crank rocker mechanism is used to transform the continuous rotation of the motors to the back and forth motion of the leg, where crank $A B$ driven by the hip motor rotates around point $A$, and linkage $B C$ transmits 
the power to rocker $C D$. Because $D C G H$ is a rigid body, the robot humerus can quickly swing back and forth.

$D E F G$ : Rocker $D E$ driven by the knee motor swings back and forth, and the motion is transmitted to the other rocker $G F$ through linkage $E F$, where $G F J$ can be considered a rigid body. Then, the robot radius $H I$ can swing quickly through the parallel four-bar linkage GHIJ.

The dimension parameters of the designed leg are shown in Tab. 1.

Table 1 Dimension parameters of leg
\begin{tabular}{|l|c|l|c|}
\hline \multicolumn{1}{|c|}{ Parameter } & Value $/ \mathrm{mm}$ & \multicolumn{1}{c|}{ Parameter } & Value $/ \mathrm{mm}$ \\
\hline Thigh $L_{D H}$ & 150 & Frame $L_{A D}$ & 130 \\
\hline Calf $L_{D H}$ & 150 & Rocker $L_{D E}$ & 20 \\
\hline Foot $L_{I K}$ & 150 & Link $L_{E F}$ & 105.5 \\
\hline Crank $L_{A B}$ & 22 & Rocker $L_{F G}$ & 32.5 \\
\hline Link $L_{B C}$ & 170.2 & Frame $L_{D G}$ & 110 \\
\hline Rocker $L_{C D}$ & 75 & & \\
\hline
\end{tabular}

In this study, the material of the designed leg is Al6061. The angle range of hip joint is $85^{\circ}-160^{\circ}$, and the angle range of knee joint is $30^{\circ}-140^{\circ}$.

To guarantee the walking stability and small undulation of the body, the foot trajectories should be smooth with a low contact impact. Thereby, the low contact impact gait planning method proposed by Wang Lipeng et al. [17] with particular attention paid to the foot motion trajectory is selected. This gait imitates the movement track of animals. The motion parameters include stride length $S$, stride height $h$ and gait cycle time $T$, as shown in Fig. 2.

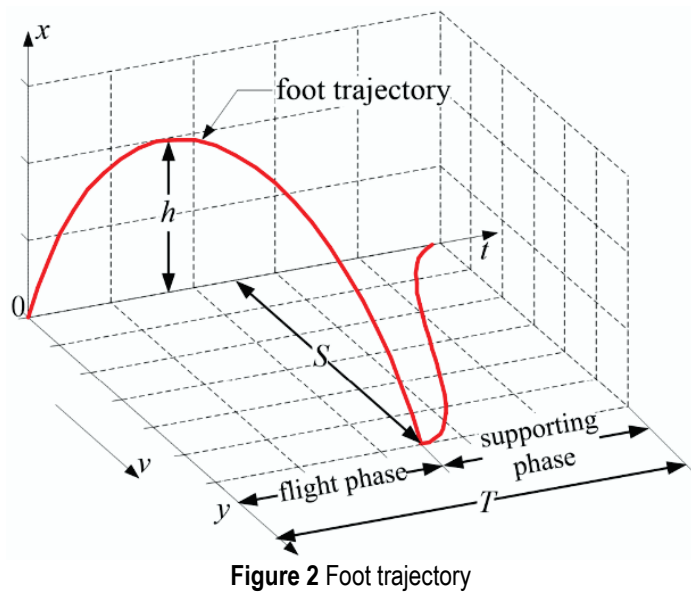

As mentioned in the paper, the knee motor drives rocker $D E$ and transmits the motion to $G F J$ and the radius $H I$, and the hip motor drives rocker $A B$ and transmits the motion to the thigh $\mathrm{DCH}$. Therefore, the robot leg motion control can be realized by accurately controlling the positions of the two joint motors. Given the foot trajectory point $K$, the angles of the hip motor and knee motor can be calculated by inverse kinematics to achieve motion control according to references [16].

\section{ESTABLISHMENT OF FINITE ELEMENT MODEL FOR FOOT-GROUND INTERACTION}

The robot studied is a quadruped robot with four legs. In view of the similarity of the trajectory of the quadruped motion, only one of the leg mechanisms is used to simulate the interaction. The robot leg is modeled in Pro/E, and then imported into the ABAQUS as shown in Fig. 3. The soil model is modeled directly in ABAQUS. Previous research has shown [18] the soil density increases rapidly firstly in the plough layer (0-200 mm depth), and then maintains an approximate stable value with no obvious changes. Therefore, the depth of the soil model is set to $200 \mathrm{~mm}$.

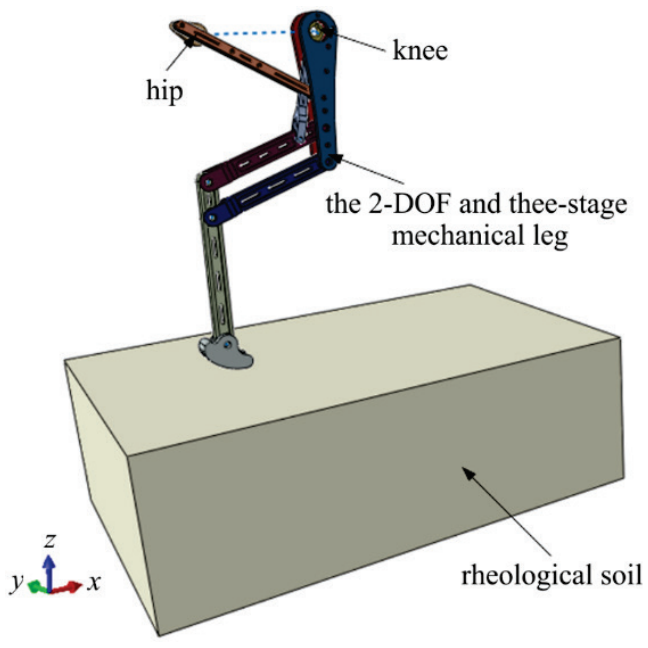

Figure 3 Assembly rendering

\subsection{Modeling of the Soil}

The farmlands in southern China have complex rheological and creep properties which can be simulated by extended Drucker-Prager (D-P) model in ABAQUS. With a non-circular yield surface on $\pi$ plane [19-20], the extended D-P model can simulate the real stretch status in three different axial. Besides, the setup model takes into consideration other factors, such as the creeping property and shear dilatancy of the soil, as shown in Fig. 4.

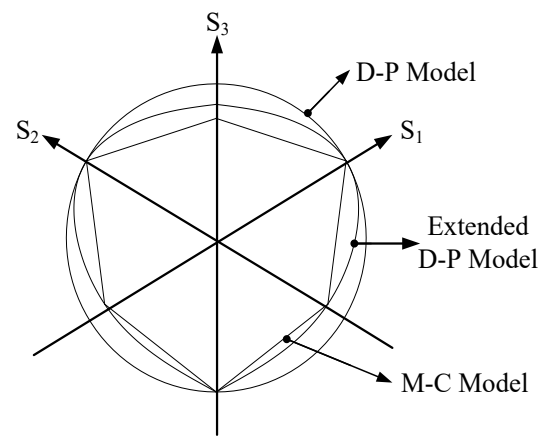

Figure 4 Yield surface on $\pi$ plane

The basic parameters of the extended D-P model includes the soil cohesion $d$, internal friction angle $\beta$, dilatancy angle $\psi$, shear modulus $G$ and flow stress ratio $K$, where $G$ can be determined by the indoor carrying-board method, $\psi=0$ because the situation is kind of the non associated flow in this paper. The cohesion $c$ and internal friction angle $\varphi$ of Mohr-Coulomb model can be measured with the shear test, and these data need to be converted into D-P parameters [19] according to Eq. (1).

$$
\begin{cases}\tan \beta=\frac{6 \sin \varphi}{3-\sin \varphi} & K=\frac{3-\sin \varphi}{3+\sin \varphi} \\ \sigma=2 c \frac{\cos \varphi}{1-\sin \varphi} & d=\left(1-\frac{1}{3} \tan \beta\right) \sigma\end{cases}
$$


The soil measured in this paper is the rheological soil of southern farmland, the specific parameters are shown in Tab. 2.

Table 2 Soil parameters
\begin{tabular}{|l|c|}
\hline \multicolumn{1}{|c|}{ Parameter } & Value \\
\hline Density $/ \mathrm{g} \cdot \mathrm{mm}^{-3}$ & 2.08 \\
\hline Compaction $/ \mathrm{N} \cdot \mathrm{cm}^{-2}$ & 117.9 \\
\hline Moisture Content $/ \%$ & 33.16 \\
\hline Poisson Ratio & 0.22 \\
\hline Cohesion $/ \mathrm{Pa}$ & $5.15 \times 10^{4}$ \\
\hline Internal Friction Angle $/{ }^{\circ}$ & 11 \\
\hline Shear Modulus $/ \mathrm{Pa}$ & $7.1 \times 10^{6}$ \\
\hline Flow Stress Ratio & 0.9407 \\
\hline
\end{tabular}

\subsection{Definition of the Mesh Type}

The walking simulation on the farmland is a multibody dynamic event belonging to a highly nonlinear problem with collision and complex contact. Since the simulation model is mainly consisted of multi-body movement and contact with large deformation, the ABAQUS explicit dynamics method is adopted in this article. Besides, the unit type is set to linear reduction integral to ensure the solving precision is not affected greatly when the deformation occurs, and the shear locking does not take place when the mesh is distorted. Considering the simulation time-consuming is proportional to the number of the mesh, and is inversely proportional to the size of the minimum mesh, the unit type of the soil model is defined as 8-node linear brick (C3D8R). The enhanced hourglass control method for the proposed contact problem is adopted where the hourglass coefficient is set to 1. Since each part of the robot leg is rigid, the 4-node linear tetrahedron (C3D4) is selected. In the grid mesh generation, the mesh size of the soil is smaller than the leg's to avoid the puncture phenomenon.

\subsection{Setting of the Contact and Boundary Conditions}

The leg basically does not deform during the movement process, so all parts of the leg are set as rigid units. The contact between them is defined as friction-free by the universal contact algorithm.

The foot should contact with the soil and produce large deformation. Hence, a "rigid - flexible contact" pair algorithm is used. The contact pair consists of a main surface and a secondary surface. The main surface is composed of rigid units, which is the foot, and the secondary surface is the upper surface of soil. The contact formula adopts finite slip. There can be any relative sliding between the two contact surfaces, and the contact pressure is calculated according to the changed contact area.

Contact properties between contact surfaces include normal action and tangential action. The tangential action is defined as penalty function, the friction factor is 0.3 , and the normal action is hard contact.

The simulation of leg movement is divided into 20 analysis steps, the type of which is set to dynamic, explicit and incremental. The time length of each analysis step is $0.05 T$, and the displacement boundary condition constraint is added to each analysis step. The displacement boundary conditions of each analysis step are obtained by Matlab software through the foot end trajectory planning and the inverse kinematics solution. Then the knee joint and hip joint motions are added to the corresponding steps to complete the driver settings of the robot leg. In addition, $5 \mathrm{~kg}$ load is applied on the robot leg to simulate the robot weight, and the leg movement is limited to move vertically. In order to simulate the real soil environment, a fully fixed constraint is imposed at the bottom of the soil, and an axial symmetry constraint is put to all around of the soil to avoid the side displacement of the soil. Finally, the gravitational field is applied to the whole model to complete the setting of the boundary condition.

\section{SIMULATION TEST \\ 4.1 Orthogonal Experiment}

The foot structure plays a vital role in the stability and energy consumption of the legged robot. The typical foot structures (arc, hemisphere, cylinder) are shown in Fig. 5.

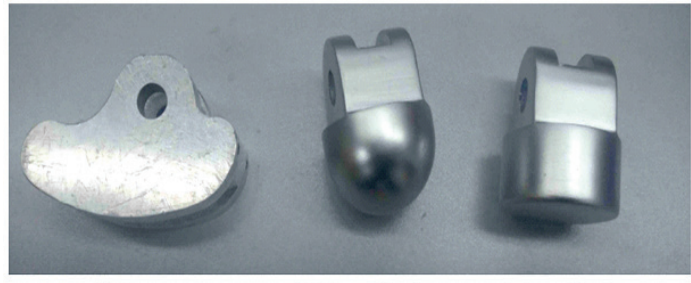

$\begin{array}{lll}\text { (a) } \operatorname{arc} & \text { (b) hemisphere } \quad \text { (c) cylinder }\end{array}$

Figure 5 Three typical foot structures

In order to reduce the energy consumption of the robot, the orthogonal experiment is conducted to find out the optimal gait parameters and foot structure. There are four factors in the experiment, which is stride length $S$, stride height $h$, gait cycletime $T$, and foot structure. Each factor is designed at 3 levels, as shown in Tab. 3.

Table 3 Orthogonal experiment factor

\begin{tabular}{|c|c|c|c|c|}
\hline No. & A & B & C & D \\
\hline Factor & $S / \mathrm{mm}$ & $h / \mathrm{mm}$ & $T / \mathrm{s}$ & Structure \\
\hline Level 1 & 300 & 70 & 1.2 & Arc \\
\hline Level 2 & 200 & 55 & 1.8 & Cylinder \\
\hline Level 3 & 150 & 40 & 2.4 & Hemisphere \\
\hline
\end{tabular}
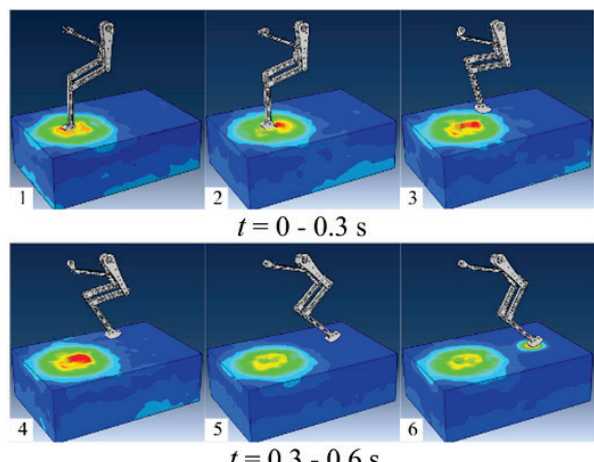

$t=0.3-0.6 \mathrm{~s}$

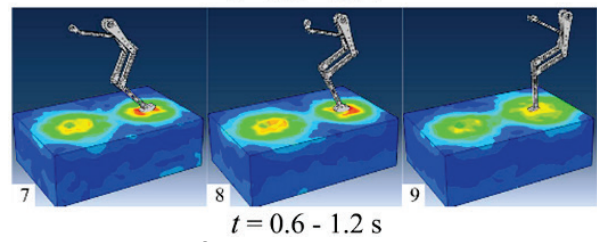

Figure 6 Simulation process renderings

Four influencing factors and three levels are investigated in this experiment, so the orthogonal test table 
$L 9\left(3^{4}\right)$ is selected. The energy consumption of the leg movement is obtained in the ABAQUS simulation of 9 samples testing. As shown in Fig. 6, the robot lifts the leg in $0-0.3 \mathrm{~s}$, gets into the flight phase in $0.3-0.6 \mathrm{~s}$, and walks within the soil in $0.6-1.2 \mathrm{~s}$ when the gait cycle time is 1.2 s. These simulation results are well consistent with theoretical results

\subsection{Experimental Analysis}

Using the energy consumption per $1 \mathrm{dm}$ as metrics, the orthogonal experiment results are listed in Tab. 4.

\begin{tabular}{|c|c|c|c|c|c|}
\multicolumn{7}{|c|}{ Table 4 The orthogonal experiment results } \\
\hline Number & A & B & C & D & Test Results \\
\hline Factor & $\begin{array}{c}\text { Stride } \\
\text { Length }\end{array}$ & $\begin{array}{c}\text { Stride } \\
\text { Height }\end{array}$ & $\begin{array}{c}\text { Gait } \\
\text { Cycle } \\
\text { Time }\end{array}$ & $\begin{array}{c}\text { Foot } \\
\text { Structure }\end{array}$ & $\begin{array}{c}\text { Energy } \\
\text { Consumption } \\
\text { Ratio } / \mathrm{J} \cdot \mathrm{dm}^{-1}\end{array}$ \\
\hline Test 1 & 1 & 1 & 1 & 1 & 562.98 \\
\hline Test 2 & 1 & 2 & 2 & 2 & 267.69 \\
\hline Test 3 & 1 & 3 & 3 & 3 & 576.55 \\
\hline Test 4 & 2 & 1 & 2 & 3 & 516.72 \\
\hline Test 5 & 2 & 2 & 3 & 1 & 204.04 \\
\hline Test 6 & 2 & 3 & 1 & 2 & 270.87 \\
\hline Test 7 & 3 & 1 & 3 & 2 & 135.75 \\
\hline Test 8 & 3 & 2 & 1 & 3 & 499.69 \\
\hline Test 9 & 3 & 3 & 2 & 1 & 230.90 \\
\hline
\end{tabular}

A range analysis of the experiment results is shown in Tab. 5. The analysis results show that the successive order of factors affecting the energy consumption ratio (ECR) is foot structure, stride length, gait cycle time and stride height.

Table 5 Range analysis of the orthogonal experiment

\begin{tabular}{|l|c|c|c|c|}
\hline \multicolumn{1}{|c|}{ Factor } & $\begin{array}{c}\text { Stride } \\
\text { Length }\end{array}$ & $\begin{array}{c}\text { Stride } \\
\text { Height }\end{array}$ & $\begin{array}{c}\text { Gait Cycle } \\
\text { Time }\end{array}$ & $\begin{array}{c}\text { Foot } \\
\text { Structure }\end{array}$ \\
\hline Average 1 & 469.1 & 405.2 & 444.5 & 332.6 \\
\hline Average 2 & 330.5 & 323.8 & 338.4 & 224.8 \\
\hline Average 3 & 288.8 & 359.4 & 305.4 & 531.0 \\
\hline Difference $R$ & 180.3 & 81.3 & 139.1 & 306.2 \\
\hline
\end{tabular}

Table 6 Variance analysis of the orthogonal experiment

\begin{tabular}{|c|c|c|c|c|c|}
\hline Factor & $\begin{array}{c}\text { Squared of } \\
\text { Deviance }\end{array}$ & $\begin{array}{c}\text { Degree of } \\
\text { Freedom }\end{array}$ & Variance & $F$ Ratio & Salience \\
\hline Step stride & 53485 & 2 & 26793 & 5.37 & \\
\hline $\begin{array}{c}\text { Stride } \\
\text { Height }\end{array}$ & 9960 & 2 & 4980 & 1.0 & \\
\hline $\begin{array}{c}\text { Gait Cycle } \\
\text { Time }\end{array}$ & 31670 & 2 & 15835 & 3.18 & \\
\hline $\begin{array}{c}\text { Foot } \\
\text { Structure }\end{array}$ & 144688 & 2 & 72344 & 14.528 & Salience \\
\hline
\end{tabular}
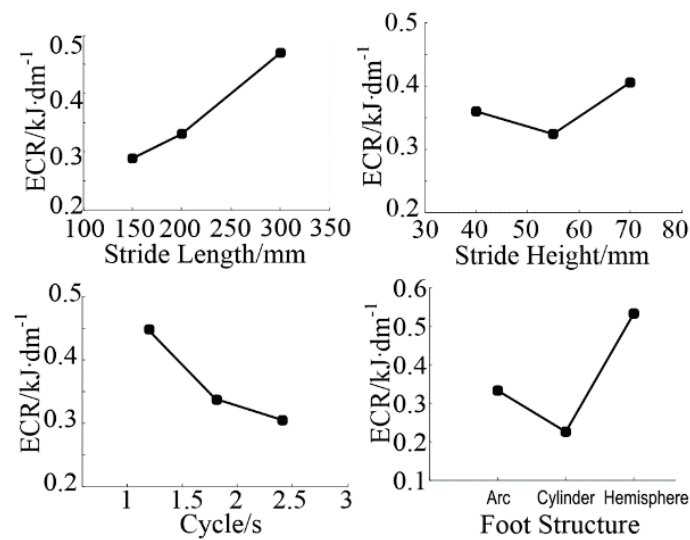

Figure 7 The influences of each experimental factor on energy consumption ratio
The results of the orthogonal experiment are analyzed by variance analysis and the significance test of each factor. In this experiment, the square of deviance of step height is considered as error term, and the significance level, the critical value. The variance analysis results of the orthogonal experiment are shown in Tab. 6. when the confidence is $90 \%$. As seen from the table, the foot structure has a significant effect on ECR.

The detailed influences of each experimental factor on ECR are shown in Fig. 7.

It is evident from Fig. 7 that the optimal combination of gait parameters and foot structure is A3B2C3D2, which means that the energy consumption in every $1 \mathrm{dm}$ is the minimum when the stride length is $150 \mathrm{~mm}$, the stride height is $55 \mathrm{~mm}$, the gait cycle time is $2.4 \mathrm{~s}$ and the foot structure is cylindrical. Using the optimal combination, the simulation experiment is carried out to obtain the relationship between the external energy consumption with time, as shown in Fig. 8.

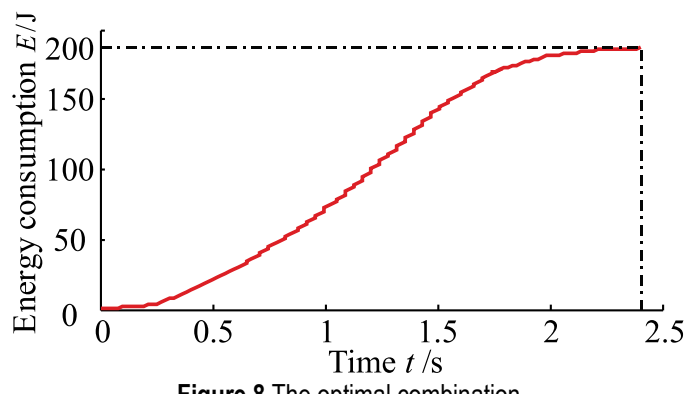

Figure 8 The optimal combination

It can be concluded that the total energy consumption in one gait cycle is $187.8 \mathrm{~J}$ when the robot moves forward the length of a stride length $(150 \mathrm{~mm})$, so the ECR is $187.8 / 1.5=125.2 \mathrm{~J} \cdot \mathrm{dm}^{-1}$. As shown in Tab. 4, the minimum ECR in the orthogonal experiment (A3B1C3D2) is 135.75 $\mathrm{J} \cdot \mathrm{dm}^{-1}$. Compared with the ECR of A3B1C3D2, the ECR of the optimal combination is $7.8 \%$ lower, which indicates the effectiveness of the orthogonal experiment.

\section{TEST ON THE SOIL BIN TEST-BED}

The soil bin test-bed of one-legged movement is developed to verify the correctness of the simulation results. The developed soil test-bed setup, shown in Fig. 9, consists of a steel soil bin of $40 \mathrm{~cm}$ width, $120 \mathrm{~cm}$ length and $30 \mathrm{~cm}$ height, filled up to $20 \mathrm{~cm}$ with soil. This soil bin is large enough to avoid side wall and bottom effects in the system level test runs for robot legs within the dimensions of the actual robot leg prototypes. Besides performing the soft soil experiment, a wooden board can be installed on the soil bin to conduct a walking experiment on a hard surface.

In this study, a high power density DC brushless motor (Maxon EC 45, 200-W) is selected as the hip motor and knee motor. The output torque can reach $15 \mathrm{Nm}$ with a GP $42 \mathrm{C}$ gear box. The EPOS2 50/5 motor driver is selected to communicate with an industrial computer using the CANopen protocol. To precisely measure the speeds and torques generated by the motor, a JN-DNJ 50 digital torque and rotational speed sensor is installed between the robot leg and the motor. 


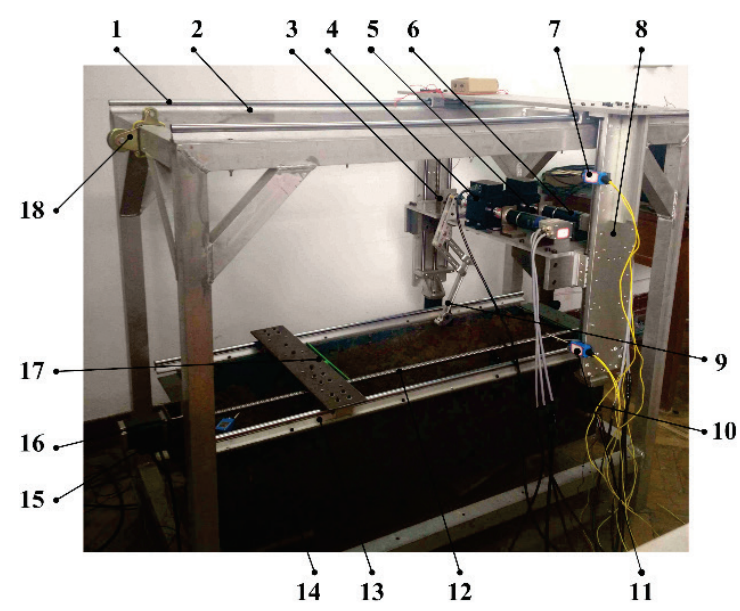

Figure 9 Soil bin test-bed for single leg: 1 - Slider, 2 - Frame, 3 - Leg brace, 4 Digital torque and rotational speed sensor, 5 - Coupling, 6 - DC Motor, 7 - Limit Switch 1, 8 - Screw slide module, 9 - Robot leg, 10 - Stepper motor 1, 11 - Limit switch 2, 12 - Ball screw, 13 - Slider, 14- Soil bin, 15 - Plummer block housing, 16 - Stepper motor 2, 17 - Soil scraper, 18 - Fixed pulley.

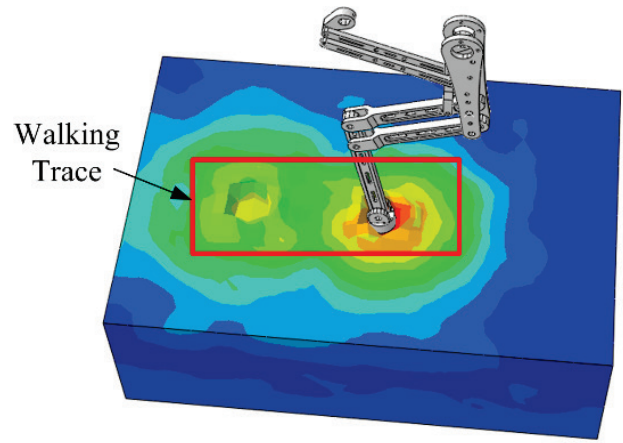

(a) Simulation test

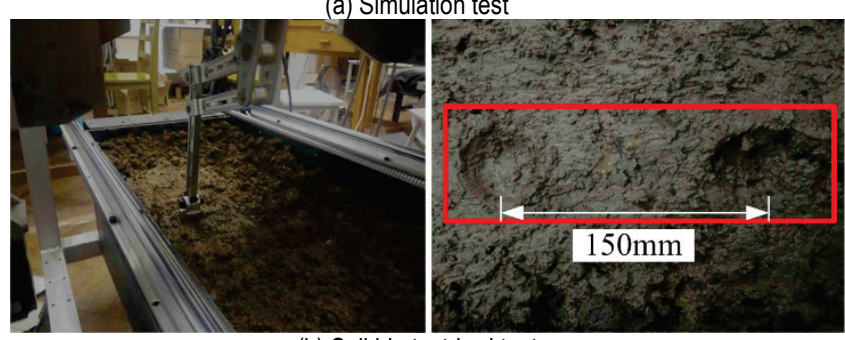

(b) Soil bin test-bed test

Figure 10 Interaction between foot and soil

The torque and rotation speed of the motor can be measure by the digital torque and rotational speed sensor on the soil bin test-bed, and then the instantaneous power can be obtained by the following Eq. (2).

$P=\frac{T \cdot n}{9550}$

where $T$ is torque $(\mathrm{Nm}), P$ is power $(\mathrm{kW}), n$ is rotational speed (r/min)

The optimum gait parameters and foot structure are adopted in the soil bin test-bed, and Fig. 10. shows the simulation results and the actual test of the interaction between the foot and the soil. As can be seen, the actual compacting effect and the distance traveled of the robot leg on the soil is coincident with the simulation results.

The data are collected and processed by PC, and the variation of torque and power in a gait cycle are obtained, as shown in Fig. 11.

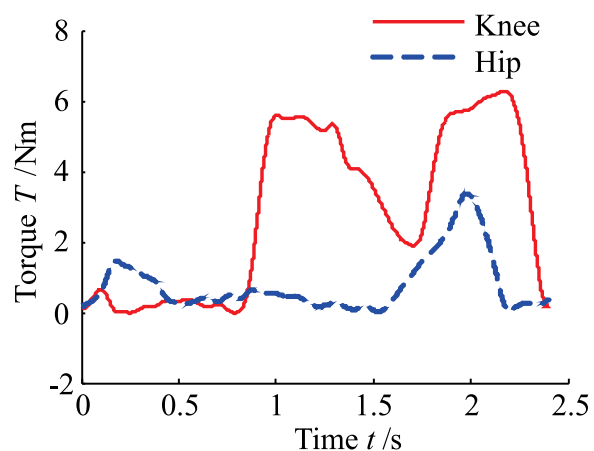

(a) Variation of joint torque

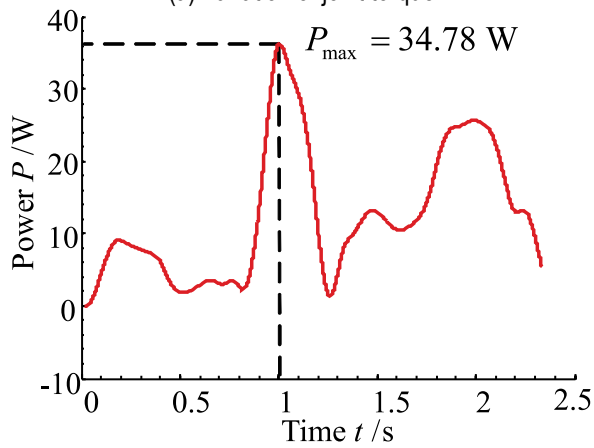

(b) Variation of system power

Figure 11 Experiment results of soil bin test-bed

Fig. 11 shows that the energy consumption increases dramatically around $1 \mathrm{~s}$, which corresponds to the process of robot leg penetrating the soil. After this process, the power decreases to 0 rapidly, and then the power increases gradually as the robot leg moves forward in 1.2-2.4 s.

In the simulation, the energy is supplied by the outside, but the energy required in the actual experiment is basically supplied by the motor, therefore, the total energy output from the motor is approximately equivalent to the total energy required. The comparison between the actual energy consumption and the simulated energy consumption in a gait cycle is shown in Fig. 12.

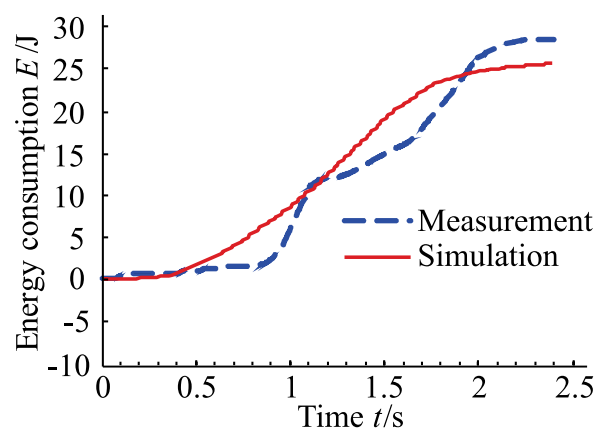

Figure 12 Energy consumption comparison between measurement and simulation

According to Fig. 12, their trend is very similar by comparing the simulation with the measuring curve. Since there are some clearance between each bar of the robot leg, reversing the motor lead to a short-term pause of the leg, thus the increase of energy consumption in the actual experiment is slowing down at $1.2 \mathrm{~s}$. Most of the energy provided by the system is used to overcome the resistance of the soil and drive the mechanism leg. The total energy consumption of the simulation test is $25.86 \mathrm{~J}$, while the test-bed is $27.94 \mathrm{~J}$. The error between them is $7.4 \%$. This tolerable error verifies the correctness and validity of the orthogonal experiment. 


\section{CONCLUSION}

In this paper, a novel electric drive robot leg based on the double four-bar mechanism is proposed, and then a FEA model for foot-ground interaction based on ABAQUS explicit dynamics analysis is established, and the optimal gait parameters and foot structure are obtained by the orthogonal experiment subsequently. The results demonstrate that the successive order of factors affecting the energy consumption ratio (ECR) is foot structure, stride length, gait cycle time and stride height. The energy consumption in every $1 \mathrm{dm}$ is the minimum when the stride length is $150 \mathrm{~mm}$, the stride height is $55 \mathrm{~mm}$, the gait cycle time is $2.4 \mathrm{~s}$ and the foot structure is cylindrical. The process of penetrating the soil consumes the most energy in a gait cycle. The optimization design of the foot structure and the penetration process is helpful to reduce the energy consumption of the farmland robot.

The established simulation system of legged robot on farmland soil is consistent with the actual environment. It can simulate the dynamic walking of a single leg on the farmland soil, analyze the energy consumption and motion characteristics, reveal the interaction mechanism between the leg and the farmland soil, and provide scientific basis for the leg design and optimization of the farmland legged robot.

In the next study, an active degree of freedom will be added to the hip joints of the robot's legs so that the penetrating process can be controlled to further reduce ECR. In addition, the effect of soil moisture content, gait type on the ECR will be explored to ensure that the robot can adapt to different environments.

\section{Acknowledgement}

This research was supported by the National Natural Science Foundation of China (grant numbers 51405239) and the Fundamental Research Funds for the Central Universities (grant numbers KY2201761).

\section{REFERENCES}

[1] Zhang, X. Y. \& Sui, Y. Y. (2005). Summarization on the effect of soil compaction on crops, Transactions of the Chinese Society for Agricultural Machinery, 36(10), 161164. Retrieved from http://en.cnki.com.cn/Article en/ CJFDTOTAL-NYJX200510042.htm

[2] Hutter, M., Remy, C. D., Hoepflinger, M. A., et al. (2011). ScarlETH: Design and control of a planar running robot, in IEEE International Conference on Intelligent Robots and Systems (IROS), San Francisco, 562-567. https://doi.org/10.1109/IROS.2011.6094504

[3] Seok, S., Wang, A., Meng,Y. C., et al. (2013). Design principles for highly efficient quadrupeds and implementation on the MIT cheetah robot, in IEEE International Conference on Robotics and Automation (ICRA). Karlsruhe, 3307-3312. https://doi.org/10.1109/ICRA.2013.6631038

[4] Cully, A., Clune, J., Tarapore, D., et al. (2015). Robots that can adapt like animals, Nature 521(7553), 503-507. https://doi.org/10.1038/nature14422

[5] Hyun, D. J., Seok, S., Lee, J., et al.(2014). High speed trotrunning: Implementation of a hierarchical controller using proprioceptive impedance control on the MIT Cheetah. The International Journal of Robotics Research 33(11), 1417-
1445. https://doi.org/10.1177/0278364914532150

[6] Maladen, R. D., Ding, Y., Umbanhowar, P. B., et al. (2011). Mechanical models of sandfish locomotion reveal principles of high performance subsurface sand-swimming. Journal of the Royal Society Interface, 8(62), 1332-1345. https://doi.org/10.1098/rsif.2010.0678

[7] Iida, M., Kang, D., Taniwaki, M., et al. (2008). Localization of $\mathrm{CO}_{2}$ source by a hexapod robot equipped with an anemoscope and a gas sensor. Computers and Electronics in Agriculture, 63(1), 73-80. https://doi.org/10.1016/j.compag.2008.01.016

[8] Aravind, K. R., Raja, P., \&Pérez R. M. (2017). Task-based agricultural mobile robots in arable farming: A review. Spanish Journal of Agricultural Research, 15(1), 1-16. https://doi.org/10.5424/sjar/2017151-9573

[9] Zhang, S., Liang, X., Xu, L., et al. (2013). Initial development of a novel amphibious robot with transformable fin-leg composite propulsion mechanisms. Journal of Bionic Engineering, 10(4), 434-445. https://doi.org/10.1016/S1672-6529(13)60247-4

[10] Ren, X., Liang, X., Kong, Z., et al. (2013). An experimental study on the locomotion performance of elliptic-curve leg in muddy terrain. IEEE/ASME International Conference on Advanced Intelligent Mechatronics (AIM). Wollongong: 518-523. https://doi.org/10.1109/AIM.2013.6584144

[11] Zhang, Q., Ding, X., \& Xu, K. (2015). Terrain adaptability mechanism of large ruminants' feet on the kinematics view. Applied Bionics and Biomechanics, 2015, 1- 9. https://doi.org/10.1155/2015/151686

[12] Zhang, Q., Xu, K., \& Ding, X. (2017). Investigation of feet functions of large ruminants with a decoupled model of equivalent mechanism. Biology Open (6), 407-414. https://doi.org/10.1242/bio.023630

[13] Ding, L., Gao, H., Deng, Z., et al. (2013). Foot-terrain interaction mechanics for legged robots: modeling and experimental validation. The International Journal of Robotics Research 32(13), 1585-1606. https://doi.org/10.1177/0278364913498122

[14] Ani, O. A., Uzoejinwa, B. B., Ezeama, A. O., et al. (2018). Overview of soil-machine interaction studies in soil bins. Soil and Tillage Research, 175, 13-27. https://doi.org/10.1016/j.still.2017.08.002

[15] Chore, H. S. \& Ingle, R. K. (2017). Soil-structure interaction analyses of pile supported building frame. ASEAN Journal on Science and Technology for Development, 25(2), 457-467. https://doi.org/10.29037/ajstd.276

[16] Zhang, Y. N., Wang, X. S., Xin, Y. H., et al. (2017). Quadruped robot leg optimization based on a multi-objective genetic algorithm. Mechanika. 23(6), 881-890. https://doi.org/10.5755/j01.mech.23.6.19854

[17] Wang, L. P., Wang, J. Z., Wang, S. K., et al. (2013). Strategy of foot trajectory generation for hydraulic quadruped robots gait planning. Journal of Mechanical Engineering, 49(1), 39-44. https://doi.org/10.3901/JME.2013.01.039

[18] Zhai, L. X., Ji, C. Y., Ding, Q. S., et al. (2013). Optimized design of plough body structural and working parameters. Transactions of the Chinese Society for Agricultural Machinery, (8), 57-62. https://doi.org/10.6041/j.issn.1000-1298.2013.08.010

[19] Payandehpeyman, J., Majzoobi, G. H., \& Bagheri, R. (2016). Determination of the extended Drucker-Prager parameters using the surrogate-based optimization method for polypropylene nanocomposites. The Journal of Strain Analysis for Engineering Design, 51(3), 220-232. https://doi.org/10.1177/0309324715627564

[20] Mir, A., Luo, X., \& Siddiq, A. (2017). Smooth particle hydrodynamics study of surface defect machining for diamond turning of silicon. The International Journal of Advanced Manufacturing Technology, 88(9), 2461-2476. https://doi.org/10.1007/s00170-016-8940-6 


\section{Contact information:}

Xiaohan WANG, Prof. Dr.

(Corresponding author)

College of Engineering, Nanjing Agricultural University,

No. 40 DianJiangtai Road, Pukou District,

Nanjing City, 210031, Jiangsu Province, China

E-mail: wangxiaochan@njau.edu.cn

Yongnian ZHANG, Lecturer, Dr.

College of Engineering, Nanjing Agricultural University,

No. 40 DianJiangtai Road, Pukou District,

Nanjing City, 210031, Jiangsu Province, China

\section{Xiaofei LUO}

College of Engineering, Nanjing Agricultural University,

No. 40 DianJiangtai Road, Pukou District,

Nanjing City, 210031, Jiangsu Province, China

\section{Cong ZHU}

College of Engineering, Nanjing Agricultural University,

No. 40 DianJiangtai Road, Pukou District,

Nanjing City, 210031, Jiangsu Province, China

Wei LU, A/Prof. Dr.

College of Engineering, Nanjing Agricultural University,

No. 40 DianJiangtai Road, Pukou District,

Nanjing City, 210031, Jiangsu Province, China 